\title{
Solving Sparse Linear Systems on NVIDIA Tesla GPUs*
}

\author{
Mingliang Wang ${ }^{1}$, Hector Klie ${ }^{2}$, Manish Parashar ${ }^{1}$, and Hari Sudan ${ }^{2}$ \\ ${ }^{1}$ NSF Center for Autonomic Computing (CAC) \\ The Applied Software System Laboratory (TASSL) \\ Rutgers, The State University of New Jersey \\ 94 Brett Road, Piscataway, NJ 08854, USA \\ \{mlwang, parashar\}@cac.rutgers . edu \\ ${ }^{2}$ ConocoPhilips \\ Houston, TX, USA \\ \{Hector.M.Klie, Hari.H.Sudan\}@conocophillips.com
}

\begin{abstract}
Current many-core GPUs have enormous processing power, and unlocking this power for general-purpose computing is very attractive due to their low cost and efficient power utilization. However, the fine-grained parallelism and the stream-programming model supported by these GPUs require a paradigm shift, especially for algorithm designers. In this paper we present the design of a GPU-based sparse linear solver using the Generalized Minimum RESidual (GMRES) algorithm in the CUDA programming environment. Our implementation achieved a speedup of over 20x on the Tesla T10P based GTX280 GPU card for benchmarks with from a few thousands to a few millions unknowns.
\end{abstract}

\section{Introduction}

Single core performance is leveling off, and recent growth has focused on on-chip parallelism and multiple cores. Chip designers are increasing processing capability by building multiple processing cores in one chip package and keep increasing the numbers of cores that can fit into a chip. Moore's Law has a new interpretation: it is the number of cores that doubles every 18 months [1]. Following this trend, commodity hardware is delivering formidable processing power at rather moderate cost and GPU is taking the lead. For example, the latest T10P GPU core embedded in a NVIDIA GTX280 graphics card delivers over a TFLOP [2], at a price tag of less than $\$ 500$. Other competing venders in graphics and general purpose computing have similar offerings.

\footnotetext{
* The research presented in this paper was supported in part by National Science Foundation via grants numbers IIP 0758566, CCF-0833039, DMS-0835436, CNS 0426354, IIS 0430826, and CNS 0723594, and by Department of Energy via the grant number DE-FG02-06ER54857, and was partially conducted as part of the NSF Center for Autonomic Computing at Rutgers University. The authors would also like to thank NVIDIA for their technical support.
} 
The huge performance available on GPUs has generated interest in using it for general purpose computing $[3,4,5]$. GPUs are stream processors and were historically designed for shader operations that involve applying a series of shader kernels to a large collection of data elements called a stream. This is a restricted form of parallelism, and while it simplifies the hardware and software required to implement it, mapping general-purpose computing to this paradigm is often difficult.

In this paper, we explore the use of GPUs for solving large sparse linear systems using the Generalized Minimal RESidual (GMRES) [6] algorithm. This is an important algorithm because many scientific applications can be transformed into solving a sparse linear system, and GMRES is the classical iterative method when the coefficient matrix is asymmetric. Our preconditioned GMRES implementation achieves a speedup of over 20x on the Tesla T10P based GTX280 GPU card, relative to a serial version ${ }^{1}$, for benchmarks with from a few thousands to a few millions unknowns. Close to the highest speedup was achieved for the largest input.

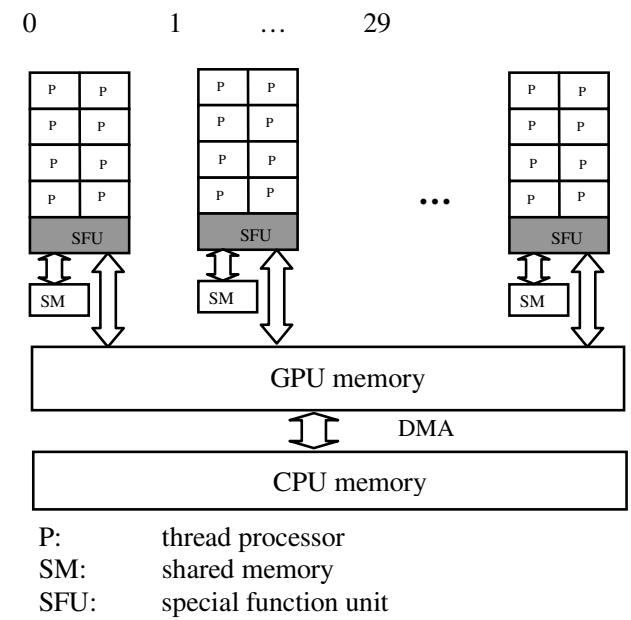

Fig. 1. Architecture overview of a Tesla T10P core for general-purpose computation

\section{Backgrounds and Related Work}

An architectural overview of the TESLA T10P GPU architecture, from the perspective of general purpose computing, is presented in Fig. 1. Note that graphics related details are omitted.

Tesla T10P SIMD core is an SIMD array of scalar processors. It has 30 multiprocessors with 8 Thread Processor (cores) each, resulting in a total of 240

\footnotetext{
1 The serial version of GMRES was based on the FORTAN version in SPARSEKIT, which was manually translated to ANSI C. The $\mathrm{C}$ version performed the same or better than the FORTRAN version for all benchmark inputs. The code was compiled using the Intel® icc version 10.1 compiler with optimization flag $-\mathrm{O} 2$, and executed on the Intel ${ }^{\circledR}$ Harpertown core clocked at $3.0 \mathrm{GHz}$. Optimization flag $-\mathrm{O} 2$ was chosen because the codes performed slightly better using - 2 as compared to using -O3 .
} 
cores. Each multiprocessor has one instruction issue unit, which broadcasts instructions to the scalar cores [2,7]. The flexibility offered by T10P cores includes the ability to execute scalar threads with arbitrary branch behaviors and memory access patterns. In [2], this exposure of the SIMD units as individual programmable scalar units is called Single Thread Multiple Thread (SIMT). However this flexibility comes at a cost if it is not exploited properly [2,7].

The GPU platform also has a more complex memory structure. In case of the T10P, cores have access to a set of off-chip memory spaces: global memory, local memory, constant memory, and texture memory. Constant and texture memory are cached but global and local memory are not. The on-chip memory includes a small shared memory (16KB on a T10P chip) that is local to each multiprocessor and can be used as software managed cache. The instruction cache is transparent to programmers. For general purpose computing on a GPU, most programmers only use global memory, shared memory, and local memory. The latency of a global memory access is about 400 core clock cycles, and that of a shared memory access is 1 cycle if there are no bank conflicts [7].

The connection between CPU memory and GPU memory is through a fast PCIe 16x point-to-point link. The transfer time for a given size data chunk can be computed as the following.

$$
t_{\text {transfer }}=t_{c}+\frac{S}{B}
$$

$t_{c}:$ the constant startup overhead. Device to host : $9.95 \mu \mathrm{s}$, host to device : $13.3 \mu \mathrm{s}$

$\mathrm{S}$ : bytes transfered

B : bandwidth. Device to host : $5.76 \mathrm{~GB} / \mathrm{s}$, host to device : $5.05 \mathrm{~GB} / \mathrm{s}$

Note that the above equations as well as the constants are derived using actual transfer times measured for a range of data sizes (1024 B to $64 \mathrm{MB}$ ).

\subsection{Support for Programming on GPU}

Programming GPUs for general purpose computing is not easy. Earlier programming models were based on shading languages such as HLSL, GLSL [8] and Cg [9], which are graphics oriented and have to be used with the OpenGL or DirectX APIs. In these models, computation was essentially organized as a sequence of shading operations (kernels) on graphics data (streams). Programmers had to make explicit calls to these graphics APIs to manage streams and launch kernel calls.

With the advent of Brook [10], direct general-purpose stream programming is supported on the GPU platform. General-purpose computation on a GPU is directly expressed as kernels acting on streams using this model. The model also eliminates the unnatural graphics API calls in the prior models. CUDA [7] added even more flexibility by allowing scattering, which enables direct and random access to DRAM associated with a GPU and makes programming on GPU almost as flexible as a CPU. Other features of CUDA include the exposure of the on-chip fast shared memory, local synchronization, and atomic operations on memory. 


\subsection{Related Work}

In the area of matrix computation, Galoppo et al. [11] studied the peak performance of a GPU dense matrix decomposition algorithm. Volkov et al. [12] also investigated the implementation of dense matrix computation and claimed to approach peak performance on the G80 series GPUs using their matrix-matrix multiply routine. In [13], Garland gave an introduction to sparse matrix algorithms on NVIDIA GPUs. Data parallel approach to GPU programming using the primitive segmented scan was presented in [14]. Boltz el al. [15] discussed an iterative solver for symmetric sparse matrix. These previous efforts focused on handling the peculiarities of the GPU platform while implementing a particular algorithm or algorithmic building block using the programming environment provided by the vendors. They showed impressive results and demonstrated the tremendous potential of GPUs as coprocessors.

GMRES is a widely used iterative method for solving large linear systems, and its parallelization has been attempted on various platforms using different approaches. In [16], a hybrid parallelized GMRES on GRID systems was presented. Compared to our method, it is a distributed memory implementation and involves computation of eigenvalues that requires more computation and does not efficiently use the GPU hardware. An earlier parallel implementation using PVM message passing was reported in [17]. It was programmed using a parallel version of basic linear algebra operations for distributed memory computers. It was designed for the linear systems derived from 5-point finite difference discretizations and exploited its particular communication patterns for efficiency. Our implementation nonetheless is for generic sparse linear systems.

Conjugate gradient is a related iterative algorithm that solves symmetric positivedefinite linear systems, and its implementation without preconditioning on an earlier NVIDIA GPU platform was reported in [15]. This implementation was based on an awkward graphics API and focused on issues that arose while mapping the algorithms to this unwieldy API. Our preconditioned GMRES solves generic sparse matrixes and has a powerful parallelized preconditioner.

\section{Parallelizing GMRES for Stream Processing Using GPUs}

A key aspect of programming applications to use GPUs involves partitioning the computation into a partition that runs on the CPU and another partition that runs on the GPU. The purpose of this partitioning is to offload selected, for example, compute intensive, portions to the GPU, while still using the CPU for other aspects such as global synchronization. Note that typically it is not beneficial to offload a complete application to the GPU due to the limitations of the stream-processing model. Stream processing is most appropriate when similar operations (kernels) are applied to a large collection of homogeneous data elements. A kernel is applied independently to each element without communication during execution. This limits the computation that can be implemented as a single kernel and therefore, most applications require a sequence of kernel calls. Additionally, an application may need to communicate with other entities or use other services, which may not be accessible from a GPU. 
Application partitioning presents several challenges. It requires programmers to manually identify which computations are appropriate for the GPU and to keep track of the computations running on the CPU and on the GPU, as well as manually initiate and manage data transfers between the two. This style of programming is not only tedious but also error-prone, and significantly impacts programmers' productivity.

To address this issue, we propose to create a high-level library that can transparently offload compute-intensive operations to GPU for certain classes of applications, and that presents, on the CPU side, a set of abstract data types as basic building blocks for programming numeric applications. These abstractions include vectors, dense matrices and sparse matrices. These data types maintain memory regions within GPU and CPU memory spaces and support synchronization between them. A set of basic operations similar to the BLAS level 1, are included. Problem specific operations such as those used in GMRES are implemented over these basic operations. The method to parallelize GMRES for the Tesla T10P GPU using CUDA is presented below.

\subsection{GMRES Iterations}

GMRES is a well-known iterative method to solve large sparse non-symmetric systems of the form $A x=b$ and was proposed by Saad and Schultz in 1986 [16]. GMRES generates a basis of dimension $m$ for the Krylov subspace $K_{m}(A, v)=\operatorname{span}\left\{v, A v, A^{2} V, \ldots, A^{m-1} v\right\}$ for a given initial residual $v$. The Krylov basis is constructed with the Arnoldi algorithm which is typically implemented in terms of the modified Gram-Schmidt orthogonalization process to generate the orthogonal basis $\left\{v_{1}, v_{2}, \ldots, v_{m}\right\}$ of the Krylov subspace $K_{m}(A, v)$.

Given the Krylov basis, an intermediate solution $x_{m}$ in the m-th iteration is given by the solution of the least squares problem: minimize $\|b-A x\|_{2}$, where $x \in x_{0}+K_{m}\left(A, r_{0}\right)$ and $r_{0}=b-A x_{0}$ is the residual associated with the initial guess $x_{0}$.

As $m$ increases, the computational cost increases at least as $O\left(m^{2} n\right)$, and memory cost increases as $O(m n)$. In large systems, this limits the largest value of $m$ that can be used. Restarted GMRES is applied to remedy this situation. After a fixed number $m$ of iterations, the solution $x_{m}$ is used as the initial guess $x_{0}$ to restart a new GMRES.

However, GMRES does not always converge, and even when it converges, it may take too many iterations to reach the desired residual tolerance. Preconditioning is a technique to improve this situation. It replaces the system $A x=b$ with the modified systems

$$
M^{-1} A x=M^{-1} b .
$$

or 


$$
A M^{-1} \hat{x}=b, x=M^{-1} \hat{x}
$$

The desired properties of $\mathrm{M}$ include 1) the modified system $M^{-1} A$ converges fast, 2) linear systems $M y=c$ easy to solve; 3) easy to parallelize. The preconditioned GMRES algorithm is shown in List 1.

The most expensive operations of this algorithm are computing the term $w=A M^{-1} v_{j}$. It requires solving a linear system with coefficient matrix $\mathrm{M}$ and a matrix-vector multiplication. In parallelizing this algorithm, we choose to find a preconditioner that makes computing the vector term $w=A M^{-1} v_{j}$ amenable to be distributed in different processors. For the time being, we do not seek parallelizing the GMRES algorithm itself.

List 1. Left-preconditioned Generalized Minimum Residual algorithm with restarts

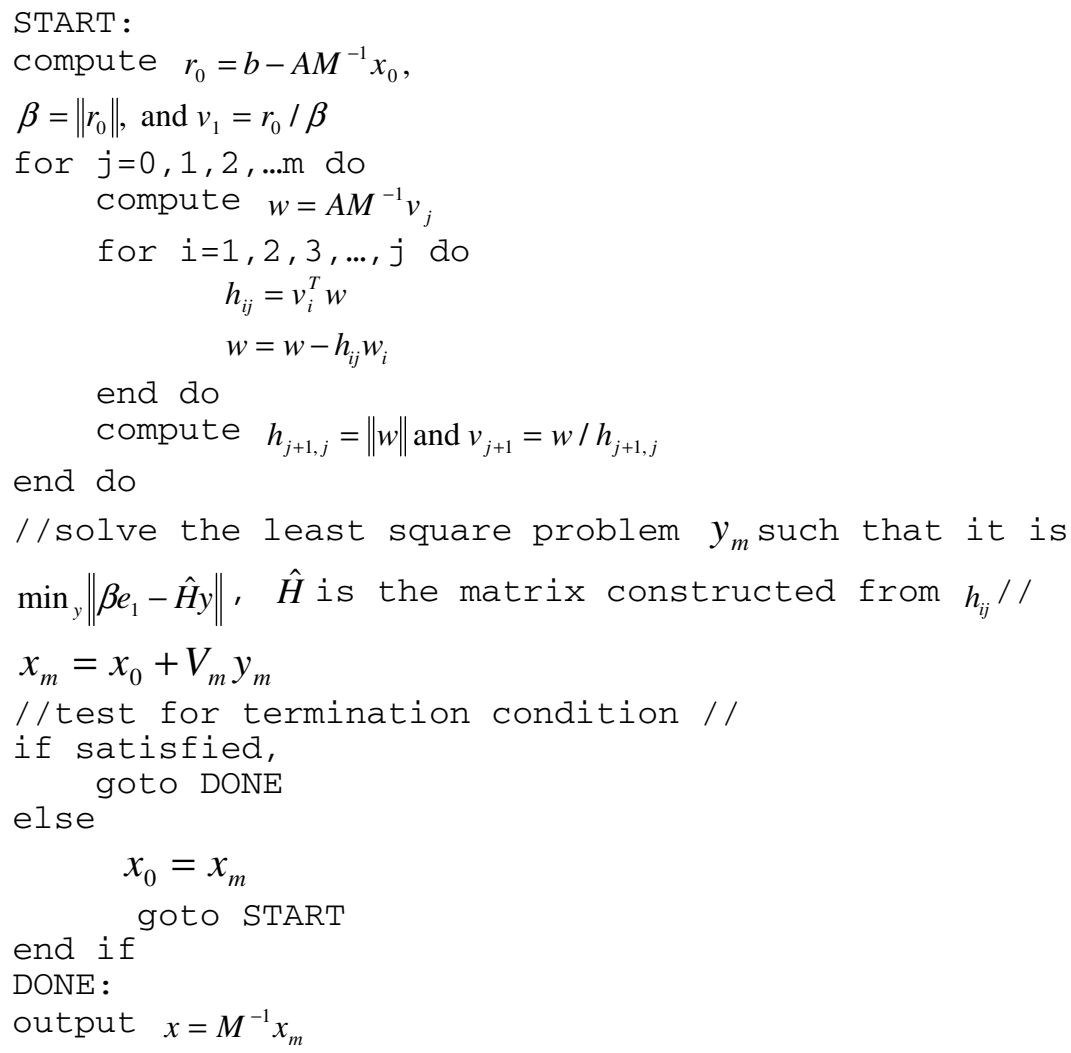




\subsection{Parallelizing Matrix Vector Multiplication and Pre-conditioning}

Sparse matrix vector multiplication appears in computing the term $w=A M^{-1} v_{j}$, and hence requires us to choose a strategy that is compatible with the parallel solve operation in the preconditioning matrix.

In the serial GMRES, incomplete LU decomposition is often used as the preconditioner as it can greatly improve the convergence rate. It is illustrated in the following equation.

$$
A=L U+E .
$$

For a sparse matrix A, it is LU-decomposed but only at the locations where A originally has non-zeros. The term $E$ accounts for the dropped elements.

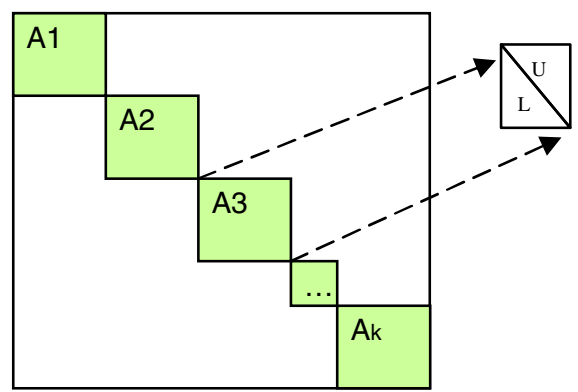

Fig. 2. Block ILU preconditioner is less effective than the conventional ILU, but has plenty of parallelism to exploit. This fits well in the data parallel model of computation on GPU. Each individual block is sparse and back/forward substitutions are used to compute $U^{-1} L^{-1} v$. The typical block size used in the experiments was 32 .

We parallelize ILU by using its variant block-ILU [19], which incurs further drops by conducting ILU only along the main diagonal. Coefficient matrix A is divided into equal sized sub-matrices and then locally decomposed using ILU, as shown in Fig. 2.

Because these blocks do not communicate to each others in decomposition and also in solving it, this scheme fits well in the data parallelism paradigm. A stream now is a collection of sub-matrices along the main diagonal.

With this parallelizing scheme for the preconditioner, we can now easily compute in parallel the sparse matrix vector multiplication by computing a lot of small matrix vector multiplication on the main diagonal blocks. General sparse matrix vector multiplication is investigated in [13].

However, the preconditioner computed from this block ILU is in general less effective than the conventional ILU computed from the whole matrix and hence requires more iterations to reach the same level of residual. 


\subsection{Polynomial Preconditioner}

A preconditioner can be enhanced by computing a polynomial of it and use this new matrix as the preconditioner. This technique is discussed in [19]. The following is a polynomial preconditioner of order $s$.

$$
M^{-1}=\left[I+N+\ldots+N^{s}\right] .
$$

with

$$
N=\left[I-\omega U^{-1} L^{-1} A\right] .
$$

We implement a polynomial preconditioner using the block ILU preconditioner and empirically compare its effectiveness.

Note that this polynomial preconditioner is not computed explicitly but through a series of forward-backward substitutions and matrix vector multiplications, built from the operations discussed in above.

\section{Experimental Evaluation}

The parallel GMRES was tested on the Tesla T10P GPU using a set of matrix data from the oil field simulation data of ConocoPhillips. The order of the system ranges from $\sim 2000$ to $\sim 1.1$ million. The serial version was run on Intel Harpertown clocked at $3.0 \mathrm{GHZ}$ and the parallel version on the same machine, with a NVIDIA GTX 280 GPU card. They were compiled using the Intel ICC version 10.1 and used CUDA SDK version 2.0. Table 1 presents the experimental results for different matrices. Residual tolerance 1e-2 reflects the actual value requested in a modeling application at ConocoPhillips.

Table 1. Experimental evaluation of GMRES on Tesla T10P GPU. Polynomial order=1, GMRES restart=20, final residual=1e-2. Times are in seconds.

\begin{tabular}{lllllllll}
\hline Matrix & $\mathrm{N}$ & $\begin{array}{l}\text { Serial } \\
\text { Iters. }\end{array}$ & $\begin{array}{l}\text { Parallel } \\
\text { Iters. }\end{array}$ & $\begin{array}{l}\text { Serial } \\
\text { Time }\end{array}$ & $\begin{array}{l}\text { Parallel } \\
\text { Time }\end{array}$ & Speedup Mem Trans. & $\begin{array}{l}\text { Diff. bewteen } \\
\text { Solutions }{ }^{2}\end{array}$ \\
\hline Sample 1 & 1946 & 3 & 6 & 0.0018 & 0.0060 & 0.3 & $5.30 \%$ & $1.8 \mathrm{e}-05$ \\
Sample 2 & 192096 & 10 & 20 & 9.41 & 1.16 & 8.1 & $2.30 \%$ & $8.0 \mathrm{e}-03$ \\
Sample 3 & 184102 & 13 & 19 & 7.67 & 0.326 & 23.5 & $3.60 \%$ & $1.3 \mathrm{e}-04$ \\
SPE 10_1 & 132000 & 40 & 40 & 4.82 & 0.70 & 6.8 & $2.70 \%$ & $2.4 \mathrm{e}-02$ \\
SPE 10_2 & 1122000 & 140 & 100 & 436.33 & 22.10 & 19.7 & $1.30 \%$ & $2.1 \mathrm{e}-03$ \\
\hline
\end{tabular}

The effect of polynomial enhancement is shown in Fig. 3, for one of the matrices. As evident in the figure, polynomial enhancement is effective, i.e., as the order of the polynomial increases, the number of iterations required to reach a desired precision decreases. However, as the order increased, each iteration requires more computation. Our empirical study showed that a low order, i.e., $1 \sim 2$, yields the best result for the all the matrices tested.

${ }^{2}$ Difference of solutions is measured as $\|\mathrm{x} 1-\mathrm{x} 2\| /\|\mathrm{x} 2\|(2$-norm), where $\mathrm{x} 1$ and $\mathrm{x} 2$ are the parallel and serial solutions respectively. 


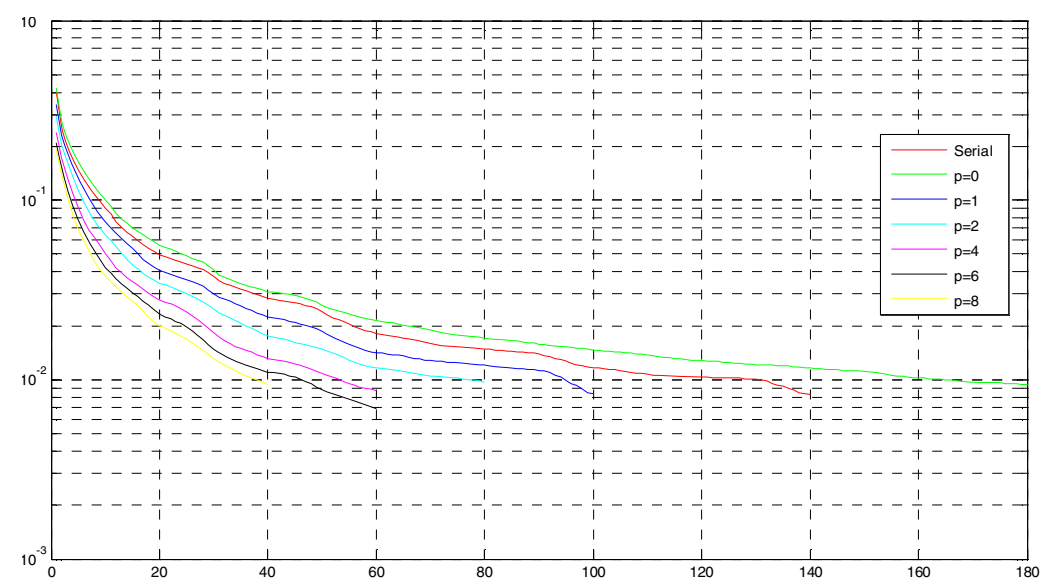

Fig. 3. Effectiveness of polynomial enhancement. Matrix $=85 \times 220 \times 60, n=1,122,000$, number of non-zero $=7,780,000$, restart $=20$.

\section{Conclusions}

The parallel implementation of GMRES on the Tesla T10P GPU presented in this paper has shown very encouraging speedups (up to $\sim 20 \mathrm{x}$ ), which confirms the great potential benefits of offloading computation to GPUs. In parallelizing GMRES, block-ILU plays a crucial role, as it not only parallelizes the preconditioning computation but also naturally decomposes the matrix-vector multiplication and the subsequent polynomial enhancement. Discovering this strategy requires deep understanding of the iterative algorithms and also the characteristics of the GPU hardware. This highlights the necessity for collaborations between domain experts and parallel program developers, as well as the need for higher-level programming support.

In the empirical study presented, it was observed that a low degree $(\mathrm{s}<=2)$ was a well-balanced choice from the perspective of effectiveness of polynomial enhancement. When the degree is larger, even though the preconditioner is still improves, the additional computation incurred dominates the gains due to reduced iterations.

In our implementation using $\mathrm{C}++$, the serial version of GMRES was around 1000 lines of codes, while the parallel one was roughly 5000 lines of code. From the productivity standpoint, this partly illustrates the additional work needed in parallel programming on GPU with CUDA.

The small C++ library developed helped hide the details of GPU programming for numerical algorithms from the applications experts and enabled the access of the GPU platform from a broader audience. 


\section{References}

1. Agarwal, A., Levy, M.: The kill rule for multicore. In: Proceedings of the 44th annual conference on Design automation, San Diego, California, pp. 750-753. ACM, New York (2007)

2. Lindholm, E., Nickolls, J., Oberman, S., Montrym, J.: NVIDIA Tesla: A unified graphics and computing architecture. Micro, IEEE 28(2), 39-55 (2008)

3. He, B., Yang, K., Fang, R., Lu, M., Govindaraju, N., Luo, Q., Sander, P.: Relational joins on graphics processors, Vancouver, Canada, pp. 511-524. ACM, New York (2008)

4. He, B., Govindaraju, N.K., Luo, Q., Smith, B.: Efficient gather and scatter operations on graphics processors. In: Proceedings of the 2007 ACM/IEEE conference on Supercomputing, Reno, Nevada, pp. 1-12. ACM Press, New York (2007)

5. Rodrigues, C.I., Hardy, D.J., Stone, J.E., Schulten, K., Hwu, W.M.W.: GPU acceleration of cutoff pair potentials for molecular modeling applications. In: Proceedings of the 2008 conference on Computing frontiers, Ischia, Italy, pp. 273-282. ACM, New York (2008)

6. Saad, Y., Schultz, M.H.: GMRES: a generalized minimal residual algorithm for solving nonsymmetric linear systems. SIAM Journal on Scientific and Statistical Computing 7(3), 856-869 (1986)

7. Nickolls, J., Buck, I., Garland, M., Skadron, K.: Scalable parallel programming with CUDA, Los Angeles, California, pp. 1-14. ACM Press, New York (2008)

8. Rost, R.J.: OpenGL(R) Shading Language. Addison-Wesley Professional, Reading (2004)

9. Mark, W.R., Glanville, R.S., Akeley, K., Kilgard, M.J.: Cg: a system for programming graphics hardware in a c-like language. In: ACM SIGGRAPH 2003 Papers, San Diego, California, pp. 896-907. ACM Press, New York (2003)

10. Buck, I., Foley, T., Horn, D., Sugerman, J., Fatahalian, K., Houston, M., Hanrahan, P.: Brook for GPUs: stream computing on graphics hardware. ACM Trans. Graph. 23(3), 777-786 (2004)

11. Galoppo, N., Govindaraju, N.K., Henson, M., Manocha, D.: LU-GPU: Efficient algorithms for solving dense linear systems on graphics hardware. In: Proceedings of the 2005 ACM/IEEE conference on Supercomputing., p. 3. IEEE Computer Society, Los Alamitos (2005)

12. Volkov, V., Demmel, J.: LU, QR and Cholesky factorizations using vector capabilities of GPUs. Technical Report UCB/EECS-2008-49, EECS Department, University of California, Berkeley (May 2008)

13. Garland, M.: Sparse matrix computations on manycore GPU's, Anaheim, California, pp. 2-6. ACM, New York (2008)

14. Sengupta, S., Harris, M., Zhang, Y., Owens, J.D.: Scan primitives for GPU computing. In: Proceedings of the 22nd ACM SIGGRAPH/EUROGRAPHICS symposium on Graphics hardware, San Diego, California, pp. 97-106. Eurographics Association (2007)

15. Bolz, J., Farmer, I., Grinspun, E., Schroder, P.: Sparse matrix solvers on the GPU: conjugate gradients and multigrid. In: ACM SIGGRAPH 2003 Papers, San Diego, California, pp. 917-924. ACM, New York (2003)

16. Zhang, Y., Bergere, G., Petiton, S.: A parallel hybrid method of GMES on grid system. In: Parallel and Distributed Processing Symposium, IPDPS 2007, IEEE International, pp. 1-7 (2007)

17. Dias, R., Cunha, D., Hopkins, T.: A parallel implementation of the restarted GMRES iterative algorithm for nonsymmetric systems of linear equations. Adv. Comp. Math 2, 261-277 (1994)

18. He, H., Bergere, G., Petiton, S.: GMRES method on lightweight grid system. In: Proceedings of the 4th International Symposium on Parallel and Distributed Computing, pp. 74-82. IEEE Computer Society Press, Los Alamitos (2005)

19. Saad, Y.: Iterative Methods for Sparse Linear Systems, 2nd edn. Society for Industrial and Applied Mathematics (April 2003) 